INDIAN JOURNAL OF POWER \& RIVER VALLEY DEVELOPMENT www.ijprvd.info

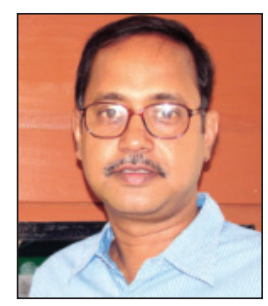

\title{
Hydrogen technology and economy: adoption challenges under the shadow of COVID-19
}

In January 2017, the Hydrogen Technology Council (“The Hydrogen Council”) was formed in Davos. Members of the Council adopted a road map of the development of hydrogen's technologies with plan to invest it. 13 parent companies of the Council showed their intentions for annual contributions at approximately $\$ 25$ billion in a year, and by 2030 for the expense of their funds in the hydrogen industry to invest US $\$ 280-300$ billion. According to the Council assessment to the 2050 annual turnover of funds in the world hydrogen energy economy will amount to $\$ 2.5$ trillion. Hydrogen Council and IRENA, both in its renewable energy roadmap reports, estimated an 18\% and 6\% hydrogen share, respectively, in total final energy consumption by 2050 . The above target will never be met if swift, decisive, and coordinated action - a prospect almost impossible to see today- is not taken to stave off the risks posed by COVID-19 and Oil \& Gas crisis.

Before the onset of COVID-19, hydrogen was used mainly in some branches of industry. In 2018, natural gas (196 million toe), coal ( 75 million toe), oil (2 million toe), electricity were used for its production. The demand for hydrogen in the world in 2018, according to the evaluations and IEA, exceeded 73 million tonnes. The hydrogen consumption

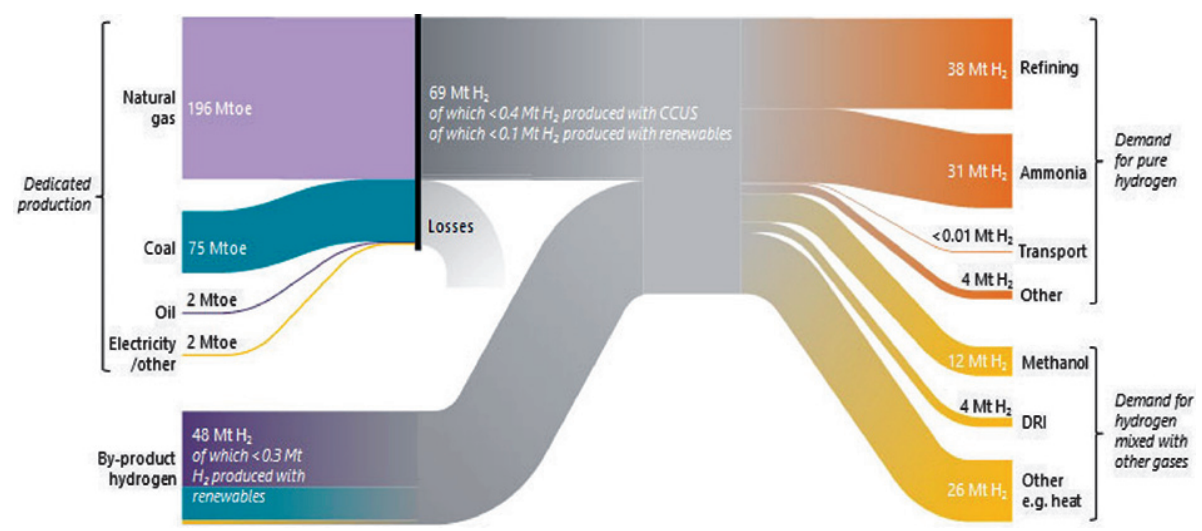
with significant impurities of other gases, as a byproduct, was about 42 million tonnes. The largest consumers of hydrogen, as can be seen from the figure, are: oil refining ( $33 \%$ of total demand); ammonia production (27\%); methanol production (11\%) and ferrous metallurgy (3\%). The demand for hydrogen in these sectors is due to its characteristics such as lightness, activity, high heat of combustion, the possibility of production and storage on an industrial scale.

Oil \& Gas industry, touted as one of the major emitters of carbon emissions, was actively involved in the energy transition before the Covid-19 pandemic, with the likes of Total, Shell, and BP involved in several large-scale green hydrogen demonstration projects in Europe. But recent oil price crash could delay such planned and under-development projects, as the oil majors work to salvage their core business in the short to medium term. Fear of global economic recession will not let the oil prices to recover in the near term from their lowest levels in the last two decades and will further undermine the competitiveness of green investments. Reduction in electricity demand would have a direct impact on the new installation. In a cohortlike example a decline in solar energy installations up to $17-48 \%$ is expected in 2020 , with solar panel production slowdowns in India, Australia, and China. In India, an 18\% reduction in wind installations and $20 \%$ cut in solar installations are being projected, as compared to the last year. In Europe, the supply chain of solar PV and wind have been disrupted. Construction activities for renewable installations have been slowed or shut down due to lockdown. Manufacturing in Italy, Spain and several other European countries has been suspended due to lockdown measures. As of now, reset of renewable and hydrogen energy in the global energy mix looks little distant. 